\title{
Lower blood pressure targets: to whom do they apply?
}

For many decades, the optimum blood pressure treatment target to prevent cardiovascular disease has been debated. Until 2013, most guidelines suggested that the general population with hypertension should be treated to achieve a blood pressure goal of lower than $140 / 90 \mathrm{~mm} \mathrm{Hg}$, but that the goal for some highrisk groups, such as patients with diabetes and chronic kidney disease, should be a blood pressure of lower than 130/80 mm Hg. In 2013, most guidelines relaxed the recommendations for high-risk patients, based on a lack of evidence, which was brought to attention after the disappointing results of the Action to Control Cardiovascular Risk in Diabetes (ACCORD) trial. ${ }^{1-3}$

In The Lancet, Xinfang Xie and colleagues ${ }^{4}$ present the results of a systematic review and meta-analysis of randomised controlled trials comparing intensive versus less intensive blood pressure-lowering treatment strategies. They used evidence from 19 trials with 44989 participants and 2496 major cardiovascular events to show that intensive blood pressure lowering is associated with reduced risk of the composite endpoint major cardiovascular events (relative risk reduction $14 \%$ [95\% Cl 4-22]), as well as stroke (22\% [10-32]) and myocardial infarction (13\% [0-24]). The mean blood pressure achieved in the intensive treatment group was $133 / 76 \mathrm{~mm} \mathrm{Hg}$, compared with 140/81 mm Hg in the less intensive treatment group. Xie and colleagues conclude that "there are additional benefits from more intensive blood pressure lowering, including for those with systolic blood pressure below $140 \mathrm{~mm} \mathrm{Hg}$ ". However, three issues need to be discussed further.

First, the most crucial decision in the design of a systematic review is which studies to include. In Xie and colleagues' systematic review, ${ }^{4}$ the authors included trials comparing different blood pressure targets. It is the most comprehensive compilation of such trials so far, but the results of the review still do not represent all the available evidence for blood pressure lowering. Other trials, in which antihypertensive drugs are compared with placebo, have also been done in patients with blood pressure lower than $140 / 90 \mathrm{~mm} \mathrm{Hg} .^{5}$

Second, this meta-analysis ${ }^{4}$ included trials with a wide variety of patient populations, including patients with hypertension alone, those with diabetes, those with chronic kidney disease, children, and elderly patients. Two trials done in elderly patients were included, both with a treatment goal blood pressure of lower than $140 / 90 \mathrm{~mm} \mathrm{Hg}$ in the intensive treatment group. Should we change treatment targets in elderly patients, solely because these two studies were included in the meta-analysis? Five of the included trials were confined to patients with diabetes mellitus. The changes of blood pressure targets for patients with diabetes mellitus in guidelines were done with knowledge of these trials, and additional placebocontrolled trials. ${ }^{6}$ Should we lower blood pressure goals in patients with diabetes mellitus, merely because of the inclusion of those four trials in this meta-analysis? In particular, does the large UK Prospective Diabetes Study trial ${ }^{7}$ in patients with diabetes, and a treatment goal blood pressure of lower than 150/90 mm Hg in the intensive treatment group, give any information supporting lower treatment goals?

Finally, the included trials comprised a wide range of achieved blood pressures. In the intensive treatment group, with a mean blood pressure of $133 / 76 \mathrm{~mm} \mathrm{Hg}$, the blood pressure achieved in these trials ranged from $118 / 75$ to $144 / 82 \mathrm{~mm} \mathrm{Hg}$. In the less intensive treatment group, with a mean blood pressure of $140 / 81 \mathrm{~mm} \mathrm{Hg}$, it ranged from $124 / 80$ to $154 / 87 \mathrm{~mm} \mathrm{Hg}$. The inclusion of trials irrespective of blood pressure achieved guarantees comprehensiveness and increases power, but also makes it difficult to judge the applicability of the results over the blood pressure range.

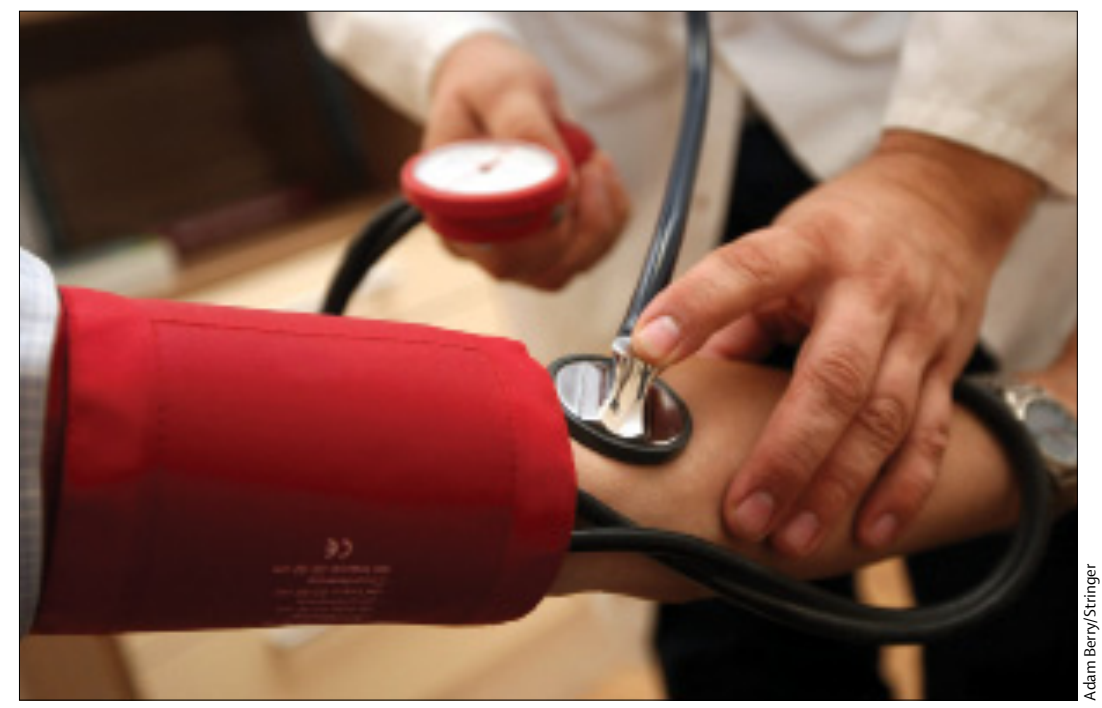

Published Online November 6, 2015 http://dx.doi.org/10.1016/ S0140-6736(15)00816-8 See Articles page 435 


\section{Comment}

In summary, Xie and colleagues' systematic review ${ }^{4}$ provides strong evidence that intensive blood pressure reduction is more beneficial than less intensive blood pressure reduction. This finding will pave the way for the treatment of a large number of additional patients compared with the number treated at present. About a third of all excess cardiovascular mortality attributable to increased blood pressure is within the normotensive range. ${ }^{8}$ Hence, with the numbers needed to treat presented by Xie and colleagues ( 94 for high-risk patients and 186 for all other included patients), this finding will be of great interest from the point of view of public health, and probably beneficial from a health economic perspective. The results of this review will probably be supported further by forthcoming results from the Systolic Blood Pressure Intervention Trial (SPRINT). ${ }^{9}$ SPRINT had a similar design to the trials included in Xie and colleagues' meta-analysis, ${ }^{4}$ with the addition of around 9000 more patients, with moderately raised blood pressure (systolic blood pressure $>130 \mathrm{~mm} \mathrm{Hg}$ ) and increased cardiovascular risk, to the 44989 analysed here. Although the evidence seems to be convincing, including studies from different populations in a meta-analysis does not mean that the overall results can be applied to all included populations. In particular, it is not yet obvious that patients with diabetes mellitus, or very elderly patients, will benefit from lower treatment targets than the recommended goal of lower than $140 / 90 \mathrm{~mm} \mathrm{Hg}$. Thus, the definition of new blood pressure treatment targets will not be an easy task, in terms of comorbidity and a specific $\mathrm{mm} \mathrm{Hg}$ target.

\section{Mattias Brunström, *Bo Carlberg}

Department of Public Health and Clinical Medicine, Division of Medicine, Umeå University, SE-901 85 Umeå, Sweden bo.carlberg@umu.se

We declare no competing interests.

1 Mancia G, Fagard R, Narkiewicz K, et al. 2013 ESH/ESC guidelines for the management of arterial hypertension: the Task Force for the management of arterial hypertension of the European Society of Hypertension (ESH) and of the European Society of Cardiology (ESC). J Hypertens 2013; 31: 1281-357.

2 James PA, Oparil S, Carter BL, et al. 2014 evidence-based guideline for the management of high blood pressure in adults: report from the pane members appointed to the eighth Joint National Committee (JNC 8). JAMA 2014; 311: 507-20.

3 Cushman WC, Evans GW, Byington RP, et al. Effects of intensive blood-pressure control in type 2 diabetes mellitus. N EnglJ Med 2010; 362: 1575-85

4 Xie X, Atkins E, Lv J, et al. Effects of intensive blood pressure lowering on cardiovascular and renal outcomes: updated systematic review and meta-analysis. Lancet 2015; published online Nov 6. http://dx.doi. org/10.1016/S0140-6736(15)00805-3.

5 Thomopoulos C, Parati G, Zanchetti A. Effects of blood pressure lowering on outcome incidence in hypertension: 2 . Effects at different baseline and achieved blood pressure levels-overview and meta-analyses of randomized trials. J Hypertens 2014; 32: 2296-304.

6 American Diabetes Association. Cardiovascular disease and risk management. Diabetes Care 2015; 38 (suppl): S49-57.

7 Stearne MR, Palmer SL, Hammersley MS, et al. Tight blood pressure control and risk of macrovascular and microvascular complications in type 2 diabetes: UKPDS 38. BMJ 1998; 317: 703-13.

8 Stamler J, Stamler R, Neaton JD. Blood pressure, systolic and diastolic, and cardiovascular risk. Arch Intern Med 1993; 153: 598-615.

9 US National Heart, Lung, and Blood Institute. Landmark NIH study shows intensive blood pressure management may save lives. Sept 11, 2015. http://www.nhlbi.nih.gov/news/press-releases/2015/landmark-nih-studyshows-intensive-blood-pressure-management-may-save-lives (accessed Nov 2, 2015).

\section{@ Late preterm rupture of membranes: it pays to wait}

Published Online November 9, 2015 http://dx.doi.org/10.1016 S0140-6736(15)00809-0

See Articles page 444
In contrast to previous assumptions, there is increasing evidence that being born in the late preterm periodbetween 34 and 36 weeks gestation-is associated with important long-term adverse effects. Several adverse outcomes have been reported, including cerebral palsy, more hospital admissions in early childhood, lower childhood height, asthma, limiting long-term illness, and poorer educational attainment. ${ }^{1-3}$ Findings from studies show a gradient of health outcomes with decreasing gestation. ${ }^{1}$ An estimated $4-5 \%$ of infants are born at 34-36 weeks, ${ }^{2,3}$ and $30 \%$ of preterm births follow prelabour rupture of the membranes. ${ }^{4}$ Because of the potential risks of fetal and neonatal infection-although with limited evidence to support this assumptionpresent guidance favours planned early delivery in women presenting with ruptured membranes at 34-36 weeks. ${ }^{5,6}$ With the emerging evidence of differences in long-term outcomes between late preterm and term infants, robust assessment of the risks and benefits of this strategy is essential, because a small increase in gestation at birth is likely to be beneficial to the infant.

In The Lancet, Jonathan Morris and colleagues ${ }^{7}$ present the results of a pragmatic randomised controlled trial of planned immediate delivery versus expectant management in women presenting with pre-labour ruptured membranes at 34-36 weeks. Findings from this trial advance substantially the evidence on the optimum management strategy in these women. 1839 women in whom there was no indication for urgent delivery were randomly assigned to immediate delivery $(n=924)$ or 